\title{
LA IMPORTANCIA DE LA INTUICIÓN MATEMÁTICA EN LOS PROCESOS DE ENSEÑANZA
}

The Importance of Mathematical Intuition in the Teaching Processes

\author{
Lina María Peña Páez ${ }^{1}$, Oscar Yesid Mariño ${ }^{2}$ \\ ${ }^{1}$ Universidad de San Buenaventura, Colombia \\ ${ }^{2}$ Universidad Distrital, Universidad de San Buenaventura, Colombia
}

KEY WORDS

Mathematical intuition

Teaching

Problem solving
ABSTRACT

This document shows how constitutive elements of intuition can lead a mathematics teacher to innovate in their educational practice. Taking into account the teaching processes in mathematics, it is proposed from the substitution method to solve integrals, to show the characteristics common to the definitions on intuition provided by both philosophers and mathematicians. We conclude that intuition not only has an important role in the epistemology of mathematics, but it can also play a fundamental role in both student learning and the teaching strategies of mathematics teachers.
PALABRAS CLAVE

Intuición matemática

Enseñanza

Resolución de problemas

\section{RESUMEN}

El presente documento muestra cómo elementos constitutivos de la intuición pueden llevar a un docente de matemáticas a innovar en su práctica educativa. Teniendo en cuenta los procesos de enseñanza en matemáticas, se propone a partir del método de sustitución para resolver integrales, evidenciar las características comunes a las definiciones sobre intuición brindadas tanto por filósofos como por matemáticos. Concluimos que la intuición, no sólo tiene un rol importante en la epistemología de las matemáticas, sino que puede jugar un papel fundamental tanto en el aprendizaje de los estudiantes como en las estrategias de enseñanza de los docentes de matemáticas. 


\section{Introducción de sección}

$\mathrm{L}$ a intuición es un concepto controvertido, aceptado por unos como fuente de conocimiento matemático $\mathrm{y}$, rechazado por otros, al considerarlo engañoso. La intuición puede entenderse como un método, al que se acude en disputas filosóficas, fundamentos de la ciencia, ética, estética, pedagogía y, a veces, en psicología. En este artículo, luego de mostrar diferentes aproximaciones a la idea de intuición matemática, se asume que la intuición es un proceso, cuyos elementos constitutivos implican, el contexto real, los conocimientos previos de quien intuye y finalmente, la necesidad de la lógica, como instrumento de validación matemática. Estos elementos, no sólo son indispensables para el matemático que inventa teorías o crea conceptos, también le son fundamentales a los docentes interesados en innovar en su práctica educativa.

Cuando de intuición matemática se trata, la literatura existente es amplia y diversa, desde las posturas filosóficas que la entienden como un criterio de certeza y verdad, quienes la comparan con la percepción en la experiencia sensorial, hasta los que la desechan por "misteriosa". Para matemáticos como Poincaré, la intuición matemática es necesaria para el proceso de invención que no sería posible si sólo se contara con los signos matemáticos o la lógica formal. Es común a muchos estudiosos del tema, entender la intuición matemática como un proceso cognitivo, que debe ser global, cuyas primeras ideas deben ser validadas por alguna teoría y que no aparecen en la mente cuando no se tienen conceptos preexistentes.

Algunos autores consideran que la intuición matemática está relacionada con esquemas estructurales del pensamiento, fundamentales en la resolución de problemas. En este artículo se asume que la intuición es necesaria para la resolución de un problema; no sólo en el ámbito de la matemática pura, es decir, se asume que un docente interesado en buscar una nueva alternativa de explicar un tema también está resolviendo un problema, en este caso un problema didáctico, y la intuición es fundamental para llegar a su objetivo.
En la primera parte se muestran diferentes posturas sobre la intuición matemática, a continuación, se estudia la relación entre la intuición y los aspectos educativos que implican, en particular, a la resolución de problemas. Finalmente se explica cómo una estrategia novedosa creada para enseñar "la regla de sustitución" en integrales, fue concebida gracias a un proceso intuitivo, teniendo en cuenta los elementos constitutivos escritos a lo largo el artículo.

\section{Aproximación a la noción de intuición matemática}

La historia de la filosofía muestra que no existe un consenso frente a la noción de intuición. Para Descartes es una operación racional total e inmediata, para Leibniz y Spinoza es una inferencia rápida para establecer los principios de la matemática. Kant, por su parte, la considera una facultad por medio de la cual se captan los objetos de conocimiento. Finalmente, para Bergson, es una forma de conocer los objetos.

En el campo de la matemática encontramos a Kurt Gödel quien considera que el papel de la intuición es análogo al papel de la percepción en el mundo de las ciencias físicas. Para Henri Poincaré la intuición es necesaria para el proceso de invención en matemáticas, más aún, es la que permite elegir el mejor camino para alcanzar el objetivo final: "necesitamos una facultad que nos muestre desde lejos el objetivo final, y esa facultad es la intuición. (Poincaré, 1905, como se citó en van Stigt, 1990, p.26).

Kitcher (1984) nos muestra cómo Kline y Cauchy asumen la intuición cómo fuente necesaria para llegar a los resultados más abstractos de la matemática.

En lo que hace referencia a las escuelas de los fundamentos de las matemáticas, encontramos a Brouwer, para quien "la intuición fue la clave para desarrollar su filosofía sistemática de las matemáticas" (van Stigt, 1990, p.134).

Para pensadores de la filosofía de las matemáticas como Penelope Maddy (1980), Charles Parsons (1980) y Elijah Chudnoff (2014), la intuición tiene que ver con el conocimiento matemático. Finalmente, tenemos a Efraim Fischbein (2002), quien considera la intuición 
como un tipo de cognición que es autoevidente, cierto, coercitivo, extrapolado, global e implícito.

De las diferentes interpretaciones sobre la intuición, aparecen dos propiedades comunes: “a) inmediatez (evidencia intrínseca) y b) certeza ( $\sin$ necesidad de demostración)" (Malaspina, 2007, p.70). Esta caracterización, contrasta con la idea de Husserl sobre la intuición, dado que, para él, las intuiciones son concluyentes en un sentido apodíctico: "las intuiciones son de los objetos (y los estados de cosas compuestos de ellos) directamente. Por su estructura (horizontal), las intuiciones se prestan a la explicación, sugieren formas en las que se pueden desplegar"(Lindström, Palmgren, Segerberg, \& Stoltenberg-Hansen, 2009, p.340).

La evolución del conocimiento ha estado enmarcada por creaciones iniciales, que en su momento han sido catalogadas como inconcebibles, absurdas, insanas o hasta insensatas, pero siempre como creaciones humanas. Los científicos saben que "la verdad no es producida por la contemplación sino por la imaginación controlada y el trabajo planificado, por la invención impaciente y el paciente ensayo de conjeturas" (Bunge, 1996, p.179).

Para Fischbein (2013) las intuiciones son cogniciones necesarias para el esfuerzo científico. Tal situación trae un problema, "por un lado, el científico necesita intuición en sus intentos por descubrir nuevas estrategias, nuevos modelos teóricos y experimentales. Por otro lado, debe ser consciente de que las intuiciones no son, como creían Descartes y Spinoza, la máxima garantía, la base principal de las verdades objetivas" (p.13). Esta situación implica que el matemático debe aprender a diferenciar entre la información objetiva y sus intuiciones impuestas subjetivamente. Esta distinción no es ni fácil, ni obvia.

Esta dualidad se ve reflejada, cuando se revisan los enunciados matemáticos, algunos de ellos (como los axiomas) parecen ser aceptados como evidentes, mientras que otros requieren de una demostración, para ser aceptados como verdaderos. Entonces las cogniciones intelectuales se pueden presentar de dos formas:

a) Una categoría de cogniciones que parecen directamente aceptables como evidentes. Estas son cogniciones intuitivas. b) Una categoría de cogniciones que se aceptan indirectamente sobre la base de una cierta prueba lógica explícita. Estas son cogniciones lógicas, o basadas en la lógica. (Fischbein, 2013, p.18)

Ahora bien, no toda cognición directa es una intuición. Las intuiciones que son cogniciones intelectuales deben expresar una situación más global, que exceda lo simplemente dado por los sentidos. En la intuición se abarcan interpretaciones, conjeturas, predicciones, soluciones y en un mayor nivel, invenciones. Tomemos el siguiente enunciado "dos cantidades iguales a una tercera son iguales entre sí", es claro, en este ejemplo, que la intuición no está, necesariamente, fundada en los sentidos.

Fischbein (2013) asume que las intuiciones son "visiones" globales y evidentes, es decir, que gracias a ellas podemos interpretar $y$ comprender de una manera global alguna situación. Sin embargo, en la vida cotidiana, la mente está acostumbrada a tratar con realidades finitas:

cuando se nos pregunta si es $0.3333 .$. . es igual a $1 / 3$ o tiende a $1 / 3$, la respuesta habitual es que 0.3333. . . tiende a 3. Para aceptar, intuitivamente, ese 0.3333 . . es igual a $1 / 3$, uno tiene que ser capaz de captar global y directamente la multitud infinita de los dígitos respectivos (Fischbein, 2013, p.33)

Por tanto, las intuiciones son la contrapartida cognitiva de algunos esquemas estructurales. Algunas veces las intuiciones pueden estar relacionadas con esquemas poco adecuados, lo que nos llevaría a intuiciones equivocadas.

Las intuiciones deben ser validadas por la lógica y la matemática formal para poder ser aceptadas por la comunidad científica, entonces "el proceso dinámico en matemáticas tiene lugar entre la intuición y la formalización, es decir, se podría decir, entre lo que podemos entender y lo que podemos expresar y comunicar, o formalizar matemáticamente de la manera habitual" (van Atten, Boldini, Bourdeau, \& Heinzmann, 2008, p.302). Aunque conozcamos todos los axiomas o las leyes de la lógica, esto no es suficiente para crear nuevas teorías, se requiere de algo más, es decir, de la intuición. Como lo afirma Poincaré (1905) la historia nos ha mostrado que "la lógica 
no es suficiente; que la ciencia de la demostración no es toda la ciencia y que la intuición debe conservar su papel como complemento, estaba a punto de decir, como contra peso o como antídoto de la lógica" (p.6).

Ahora bien, la intuición es generada por la experiencia; por las situaciones prácticas en las que el individuo está inmerso. Estas situaciones son sistemáticas y necesitan "representaciones y evaluaciones anticipatorias, globales y bien estructuradas. Una intuición puede entonces describirse como un conjunto de expectativas bien estabilizado con respecto a ciertas situaciones" (Fischbein, 2002, pp. 204-205).

Las intuiciones necesarias para resolver un problema o inventar una nueva teoría requieren que el estudiante o el matemático estén inmersos en el mundo real o abstracto de la matemática. Las ideas no aparecen de la nada, como revelaciones divinas. Los sujetos deben tener algún tipo de experiencia en el tema, sin importar el grado de conocimiento. Para Dewey la filosofía y las matemáticas se deben 'mirar siempre en el contexto de la experiencia', desmitificando el razonamiento deductivo, el conocimiento se vuelve humano, deja de ser inmutable e indudable, "y mientras que la prueba deductiva se vuelva humana y no divina o infalible, el razonamiento y la intuición no deductivos plausibles reciben su merecido como fuente de conocimiento en matemáticas, al igual que en cualquier otra parte de la vida humana"(Hersh, 2011, p.48).

Que una intuición sea correcta o no, depende de la comparación entre las intuiciones basadas en la experiencia personal del individuo y de la aceptación por parte de la comunidad científica. En el salón de clase la autoridad para determinar si una intuición es correcta o no, puede ser el profesor o el libro de texto. Las intuiciones deben irse "ajustando" de acuerdo a los nuevos esquemas que el individuo adquiera ya sea por la edad o por sus nuevos conocimientos. Veamos un ejemplo:

Las personas solían afirmar que "la multiplicación hace más grande" y la "división que hace más pequeña". Es un reclamo intuitivo basado en la experiencia temprana del niño con números naturales. Mientras se considere el conjunto de números naturales, las oraciones anteriores y las intuiciones respectivas son correctas. Pero cuando un estudiante universitario hace la misma afirmación, uno tiene una intuición incorrecta porque se debe suponer que el estudiante aprendió mucho antes sobre fracciones menores que 1, por lo que las afirmaciones anteriores (intuiciones) ya no son correctas. (Fischbein, 2013, p.35)

Sólo son posibles las intuiciones correctas cuando los esquemas mentales sean ajustados a los nuevos conocimientos, así mismo, en un proceso educativo, las intuiciones tanto del estudiante como del profesor parten de su experiencia real, en el caso del anterior ejemplo, se parte del cuerpo de conocimientos sobre los números racionales.

\section{La intuición y resolución de problemas}

La intuición matemática tiene una estrecha relación con la resolución de problemas. Para matemáticos como Poincaré (1944) la intuición es la fuente de la invención en matemáticas, para Fischbein (2013) es la base de los esquemas mentales necesarios para la construcción de la ciencia y para el aprendizaje de los estudiantes. La invención es tener una idea novedosa que permite llegar a un nuevo conocimiento, en este sentido, inventar implica resolver un problema. Todo matemático, profesor o estudiante está buscando solucionar un problema, de acuerdo a su interés o contexto.

Para Poincaré (1905) tanto la lógica como la intuición tienen un papel indispensable: "la lógica, la única que puede dar certeza, es el instrumento de la demostración; la intuición es el instrumento de la invención" (p.7). Cuando un matemático, un profesor o un estudiante quieren alcanzar una meta propuesta, requieren una guía, la cual puede ser una analogía. Cuando un inventor desea emprender la resolución de un nuevo problema, recurre a buscar problemas similares ya resueltos por el mismo método, "entonces debe percibir de qué manera se diferencia esta nueva pregunta de las de problemas anteriores y de ahí deducir las modificaciones necesarias para aplicar el método" (p.8). Cuando las analogías y diferencias 
no son muy evidentes se requiere de una penetración poco común, esto quiere decir, que el inventor, sin la ayuda de los sentidos ni de la imaginación debe "tener una comprensión directa de lo que constituye la unidad de la pieza del razonamiento, de lo que hace, por así decirlo, su alma y su fundamento" (p.8).

Para el autor francés son poco comunes aquellos analistas que pueden inventar sin la ayuda de la imaginación, que son capaces de percibir a simple vista el plan general de un edificio lógico, sin la intervención (aparente) de los sentidos. Sin embargo, "no deja de ser cierto que la intuición sensible es en matemáticas el instrumento más habitual de invención" (Poincaré, 1905, p.9). En su ensayo "invención matemática" Poincaré explica el surgimiento de su idea sobre las funciones fuchsianas, este relato sirve para comprender cómo el rigor se convirtió en el legitimador de las conquistas de la intuición. Para él, en la invención de esta idea debía estar ocurriendo 'algún tipo de pensamiento subconsciente', dando paso a la posibilidad de que el subconsciente pueda ser más inteligente que la mente consciente. Otros autores, entre los que se cuenta Hadamar (un matemático brillante) otorgan un papel primordial al subconsciente en el descubrimiento matemático, dado que éste es más desinhibido, imaginativo y creativo, lo que los lleva a relacionar al subconsciente con la intuición.

Poincaré (1905) insistirá en la necesidad de la intuición en la enseñanza de las ciencias, para él este es un comienzo en la comprensión de la matemática, más aun, aprendería a amarla, dado que sin ella "nunca llegarían a ser capaces de aplicar las matemáticas" (p.6) y si es útil para los estudiantes, lo es mucho más para el científico creativo.

Ahora bien, algunos han relacionado la "habilidad" de invención con la buena memoria o con una capacidad de atención prodigiosa. Si bien son capacidades necesarias, no son suficientes para el trabajo creativo. En realidad, la matemática no es una lista de silogismos, tiene que ver con el orden determinado en que son colocados, siendo este orden más importante que los elementos mismos.
Ahora bien, la intuición matemática es un modo general de conocimiento, estrechamente relacionada con los conceptos usados para hacer referencia al mundo físico (Ponte, 2007), lo que implica que los símbolos son la forma de comunicar lo encontrado por la intuición, a su vez, que los preconceptos son necesarios para estructurar la intuición.

Por ejemplo, cuando los estudiantes están intentando resolver un problema de máximos y mínimos, allí usan mecánicamente, los criterios de la primera y segunda derivada, sin embargo, se observa que "no han desarrollado una actitud científica que conjugue la intuición, la conjetura, la formalización y el rigor ante otros problemas de optimización" (Malaspina, 2007, p.367). Lo que significa que la intuición está relacionada con la habilidad para conjeturar y formalizar, actos que se pueden estimular a través de la resolución de problemas.

Las habilidades propias para llevar a cabo un proceso intuitivo no son exclusivas de los estudiantes, también deben ser desarrolladas por los docentes. Se debe prestar atención en "las similitudes, la capacidad de identificar isomorfismos y describir estructuras comunes. Como lo han demostrado Poincaré y Polya, las analogías entre entidades matemáticas aparentemente muy diferentes sugieren con frecuencia nuevas ideas fructíferas" (Fischbein, 2002, p.209). Muchos de los problemas didácticos podrían resolverse si se considera la importancia de la intuición en la enseñanza y aprendizaje de los estudiantes.

\section{Una aplicación de la intuición en los procesos de enseñanza de la matemática}

Para los procesos de enseñanza y aprendizaje, es necesario que el profesor integre los aspectos intuitivos y los formales para la comprensión y resolución de problemas (Solis, 1984, CasasRodriguez, 2013; Nicoletti, 2016). El docente debe controlar las fuerzas intuitivas del estudiante, pero a la vez debe incentivarlas, para que los resultados obtenidos estén acordes con el cuerpo axiomático de la matemática. Ahora bien, es importante que los profesores también tengan en cuenta los aspectos intuitivos en la 
preparación de sus clases para comprender la manera como sus estudiantes "accederán" al conocimiento (Peralta, 1995; Gómez-Chacón, 2000; Lopéz, 2006).

En su proceso de búsqueda de nuevas estrategias para lograr la aprehensión de los temas de clase, por parte de los estudiantes, el docente debe aprender a analizar y a formalizar sus adquisiciones intuitivas, "esto implica aprender a abstraer estructuras formales de realidades prácticas e interpretaciones intuitivas y cómo describirlas explícitamente" (Fischbein, 2002, p.209, p.207). El docente, muchas veces, en su práctica educativa, se enfrenta a problemas tales como: el estudiante encuentra inútil el concepto que está siendo explicado, o, no comprende la teoría o los ejercicios explicados en clase, "una de las tareas fundamentales de la educación matemática, como se ha enfatizado con frecuencia en el presente trabajo, es desarrollar en los estudiantes la capacidad de distinguir entre sentimientos intuitivos, creencias intuitivas y convicciones formales" (Fischbein, 2002, p.209, p.209).

Ahora bien, el docente debe ser el primero en identificar dicha distinción e intentar realizar actividades que conlleven a comprender esa relación. Una manera de hacerlo es a través de su propia experiencia, es decir, en el diseño de un ejercicio de clase que le permita "vivir" un proceso intuitivo que conduzca a una situación formal avalada por las proposiciones matemáticas estipuladas.

Como hemos sostenido a lo largo de este artículo, la resolución de problemas está vinculada con la intuición. Bajo esta mirada, cuando el profesor prepara su clase e intenta que sus estudiantes comprendan y apliquen los conceptos propios de la matemática; está resolviendo un problema. Fischbein (2013) propone las siguientes etapas necesarias en el esfuerzo de resolver un problema:

a) Primero, uno trata de comprender de manera significativa la pregunta dirigida, haciendo uso de la información que se muestra en el texto del problema. El solucionador debe entender claramente y distinguir lo que se da y lo que se requiere. b) En segundo lugar, el solucionador ha movilizado varias informaciones $\mathrm{y}$ asociaciones previamente adquiridas, de manera tal que se pueda salvar la brecha entre lo que se da y lo que se requiere. Tal esfuerzo mental es a veces tácito, a veces consciente, explícito. c) Cuando este esfuerzo llega a un final bien estructurado, el solucionador siente que ha alcanzado la solución. (pp.133-34)

La mencionada descripción, involucra la idea que hemos sostenido en este artículo: la intuición es un proceso dinámico, que parte del contexto real y una vez validada por el cuerpo de conocimientos de la matemática, vuelve al "mundo real" para ser aplicada y permitir, en este caso, la resolución de un problema.

A continuación, se explicará cómo a partir de la intención de un profesor por resolver un problema didáctico y su posterior aplicación, el profesor, sin ser consiente experimentó una intuición (enmarcada bajo las características, aquí descritas anteriormente).

Las tres etapas enunciadas por Fischbein involucran las características que se le han dado a la intuición, a saber, comprender de manera significativa la pregunta dirigida, implica que la intuición parte del contexto real del docente. Que el docente movilice varias informaciones $y$ asociaciones previamente adquiridas, involucra la necesidad de conceptos previos tanto matemáticos como didácticos. Si el esfuerzo llega a un final bien estructurado, significa que la solución del problema planteado cumple con el cuerpo lógico de la matemática, es decir, ha sido validada. Y el conjunto de todas estas etapas evidencia un proceso dinámico.

En la primera etapa el docente tiene una "preocupación", desea resolver un problema que le ha surgido en su práctica profesional, en su contexto real: desarrollar una nueva estrategia para explicar "la regla de sustitución" en un curso de cálculo integral.

El docente debe recurrir a los conceptos previos, que en este caso corresponden, a estrategias análogas usadas para explicar otros temas y a los conceptos necesarios para comprender la regla de sustitución. El docente, por un lado, reconoce que usar diagramas de árbol ha sido una estrategia muy útil cuando se explican temas de probabilidad. Por otra parte, conoce la importancia de las funciones compuesta y de la regla de la cadena, tema 
fundamental, para comprender la regla de sustitución. Así, moviliza y asocia informaciones tanto didácticas como matemáticas para diseñar su estrategia de aprendizaje.

Finalmente, crea su estrategia y la pone a prueba. Su esfuerzo será existo en la medida que su estrategia del árbol funcione para la mayor cantidad de integrales que debe ser resueltas usando el método de "sustitución".

Supongamos que se desea resolver la siguiente integral indefinida: $\int \frac{\operatorname{sen}(x)}{\cos ^{2}(x)} d x$.

En primera instancia, el estudiante debe reconocer todas las funciones y operaciones que aparecen implicadas en la expresión algebraica que se desea integrar. A continuación, elabora un diagrama de árbol con esta información y su conocimiento previo sobre la jerarquía de las funciones compuestas. Ver Fig. 1.

Figura 1. Diagrama de árbol para la regla de sustitución.

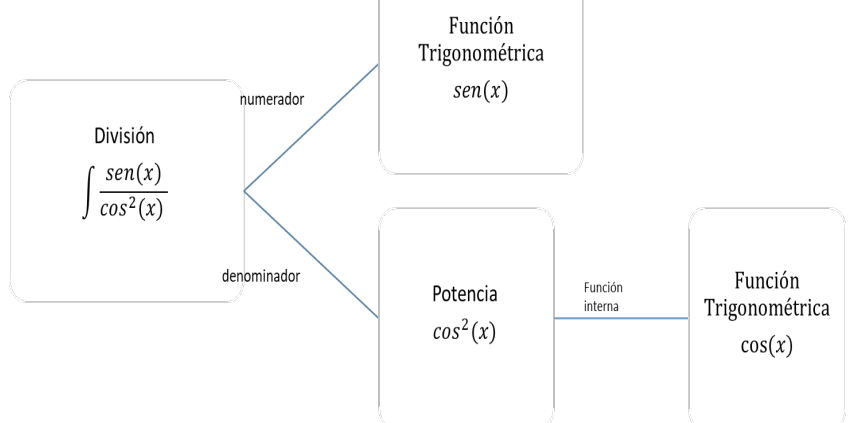

Fuente: Autores.

La última rama del árbol indica cuál es la sustitución que el estudiante debe hacer.

Entonces:

$$
u=\cos (x) \quad d u=-\operatorname{sen}(x)
$$

$$
\int \frac{\operatorname{sen}(x)}{\cos ^{2}(x)} d x=-\int \frac{d u}{u^{2}}
$$

La cuál es una integral mucho más fácil de resolver y que aparece en las tablas de las integrales.

Esta manera de enseñar la regla de sustitución no aparece en los libros comúnmente utilizados en las clases de cálculo integral. Es una idea novedosa, creada por el docente interesado en buscar una manera alternativa de lograr, por parte de sus estudiantes, una mejor compresión de la mencionada regla de integración.
El docente no llegó a esta estrategia de una manera misteriosa, tuvo que, como lo afirma Poincaré, pensar en el problema por mucho tiempo, probar diferentes formas, indagar por métodos de enseñanza de otros temas, es decir, usar analogías. Esta solución no fue una iluminación que surgió de la nada. ¿Por qué no se les ocurrió a otros profesores? ¿Acaso no están involucrados los mismos conceptos cuando se explica esta regla? Los profesores conocen los diagramas de árbol, entonces ¿por qué no encontraron la relación? Podría concluirse que ¿Algunos docentes son más competentes que otros? La respuesta es no. El asunto es el mismo que hemos mencionado anteriormente, es la lucha entre el formalismo e intuicionismo. Es la lucha entre la lógica y la intuición. Conociendo todos los símbolos matemáticos no necesariamente se llega a la creación de nuevas teorías o conceptos, se requiere ese algo adicional que es la intuición.

Pero la intuición no es una idea misteriosa que llega la cabeza de unos cuantos elegidos. Es un proceso, es algo en lo que el docente, el estudiante o el matemático, están inmersos, es algo en lo que están interesados, es decir, tiene la intención de resolver un problema. Sería poco creíble que una persona que nunca ha dado una clase de matemáticas ni tomó algún curso de cálculo infinitesimal, pudiese llegar a la estrategia didáctica, planteada en este documento.

\section{Conclusiones}

La intuición matemática es una noción ampliamente estudiada por filósofos, matemáticos, pedagogos, psicólogos y pensadores de la ciencia. Sin embargo, no existe una definición exacta con características precisas que sea acepada por toda la comunidad académica. La riqueza de sus interpretaciones en cuanto a su papel en la formación de conceptos, en la invención de nuevas teorías y en su influencia en el aprendizaje de la matemática; es un tema vigente con resultados muy positivos en la filosofía y educación de las matemáticas.

Estudios sobre la intuición permiten evidenciar algunas características comunes. La intuición matemática no es una facultad 
misteriosa propia solamente de mentes excepcionales, sino que, al ser un proceso, puede ser fortalecida por cualquier persona, es decir, que requiere de un esfuerzo. Si el esfuerzo es un esfuerzo intelectual que va más allá de la simple memoria, la compresión de símbolos matemáticos y de leyes de la lógica, entonces se llegará a la invención. En el desarrollo de la intuición intervienen los preconceptos adquiridos en la experiencia, en el contexto real de quien intuye, y a su vez, es necesaria la validación de los resultados obtenidos al final del proceso.

Quien experimenta un proceso intuitivo, llegó a este punto porque tiene una intención, es decir, desea resolver un problema. Entonces, encontramos que existe una estrecha relación entre la intuición y la resolución de problemas: sin la intuición no podría resolverse un problema. Dejando de lado el ámbito de la matemática pura, encontramos al docente, quien está interesado en resolver un problema didáctico.

Un docente interesado en innovar en su práctica educativa se encuentra enfrentado a un tipo de problema similar al del matemático que desea encontrar una nueva teoría. $\mathrm{Su}$ preocupación surge en su contexto real, a saber, su quehacer docente. Así mismo, pensar en su intención, requiere de su experiencia en la enseñanza de otros temas y de conocimientos matemáticos previos. Una vez diseñada su nueva estrategia de enseñanza, sus resultados deben ser validados e incorporados, nuevamente, al contexto del que salió su preocupación inicial.

El resultado de la intuición matemática en este trabajo está representado por el diseño de un diagrama de árbol. Este diagrama le permite al estudiante encontrar la sustitución adecuada para resolver una integral. El docente relaciona los diagramas de árbol utilizados en ejercicios de probabilidad con la composición de funciones para elaborar un diagrama de árbol cuyas ramas indican la composición de funciones y los nudos la expresión matemática correspondiente. Al final, esta técnica ayuda al estudiante a identificar más fácilmente la sustitución que debe hacer para resolver una integral indefinida. 


\section{Referencias}

Bunge, M. (1996). Intuición y razón. Buenos Aires: Editorial Sudamericana.

Casas-Rodríguez, M. (2013) Lo intuitivo como aprendizaje para el desarrollo de la actividad creadora en los estudiantes. Humanidades médicas, 22-37.

Chudnoff, E. (2014). Intuition in Mathematics. In O. Lisa M. \& H. Barbara S. (Eds.), Rational Intuition: Philosophical Roots, Scientific Investigations (pp. 174-191). https://doi.org/10.1017/CB09781139136419.010

Fischbein, E. (2002). Intuition in Science and Mathematics (Vol. 5). https://doi.org/10.1007/0-306-47237-6

Fischbein, E. (2013). Intuitions and Schemata in Mathematical Reasoning. Forms of Mathematical Knowledge, (1968), 11-50. https://doi.org/10.1007/978-94-017-1584-3_2

Gómez-Chacón, I. (2000). La intuición en Matemáticas. EDUCAR, 3 (7), p. 30-34.

Hersh, R. (2011). Mathematical Intuition (Poincaré, Polya, Dewey). Montana Mathematics Enthusiast, $8(1 / 2), 35-49$.

Lindström, S., Palmgren, E., Segerberg, K., \& Stoltenberg-Hansen, V. (Eds.). (2009). Logicism, Intuitionism, and Formalism. https://doi.org/10.1007/978-1-4020-8926-8

López, C. (2006). La intuición y la matemática. Revista de Ciencia y Tecnología Facultad de Ingeniería. Universidad de Palermo, 6, 29-36.

Maddy, P. (1980). Perception and Mathematical Intuition. The Philosophical Review, 89(2), 163-196.

Malaspina, U. (2007). INTUICIÓN , RIGOR Y RESOLUCIÓN DE PROBLEMAS. Revista Latinoamericana de Investigación En Matemática Educativa, 10(3), 365-399.

Nicoletti, J. (2016). Fundamento y construcción del acto educativo. Docencia e Investigación, 46-53.

Parsons, C. (1980). Mathematical Intuition. Procedings of the Aristotelian Society, 80, 145-168.

Peralta, J. (1995) Principios didácticos e históricos para la enseñanza de las matemáticas, Madrid, Huerga y Fierro

Poincaré, H. (1905). La intuición y la lógica en las Matemáticas. In El valor de la ciencia (pp. 1-9). Retrieved from http://casanchi.com/ref/logicaintuicion01.pdf

Poincaré, H. (1944). Invención Matemática. In Ciencia y Método (1st ed., pp. 40-54). Buenos Aires: EspasaCalpe.

Ponte, M. (2007). El papel de la intuición en la filosofía de las matemáticas. La propuesta de Charles Parsons. Laguna, (20), 35-48.

Solís, R. (1984) Ideas intuitivas y aprendizaje de las ciencias, Enseñanza de las Ciencias, 2, 83-91.

van Atten, M., Boldini, P., Bourdeau, M., \& Heinzmann, G. (2008). One Hundred Years of Intuitionism (19072007). The Cerisy Conference (1st ed.; M. van Atten, P. Boldini, M. Bourdeau, \& G. Heinzmann, eds.). https://doi.org/10.1007/978-3-7643-8653-5

van Stigt, W. P. (1990). Brouwer's Intuitionism. Amsterdam: Elsevier Science. 